\title{
Obtaining malignant melanoma indicators through statistical analysis of 3D skin surface disruptions
}

\author{
Yi Ding ${ }^{\mathrm{a}}$, Lyndon Smith ${ }^{\mathrm{a}}$, Melvyn Smith ${ }^{\mathrm{a}}$, Jiuai Sun ${ }^{\mathrm{a}}$, Robert Warr ${ }^{\mathrm{b}}$, \\ ${ }^{a}$ Machine Vision Laboratory, University of the West of England, Bristol, BS16 1QY, UK \\ ${ }^{b}$ Pigmented Lesion Clinic, North Bristol NHS Trust, Bristol, BS16 1LE, UK
}

Address of corresponding author:

Dr Lyndon Smith

Machine Vision Laboratory

Faculty of Computing, Engineering and Mathematical Sciences

DuPont Building

University of the West of England, Bristol

Frenchay Campus

Bristol

BS16 1QY

UK

Tel: +44 (0)117328 2009

Email: Lyndon.Smith@uwe.ac.uk 


\begin{abstract}
Background/purpose: It has been observed that disruptions in skin patterns are larger for malignant melanoma (MM) than benign lesions. In order to complement the classification results achieved for 2D skin patterns, this work intends to investigate the feasibility of lesion classification using 3D skin texture, in the form of surface normals acquired from a previously-built six-light photometric stereo device.

Material and methods: The proposed approach seeks to separate malignant melanoma from benign lesions through analysis of the degree of surface normal disruptions in the slant and tilt direction. A 2D Gaussian function is used to simulate a piece of healthy skin for comparison with a lesion's existing surface normals in the tilt direction and slant direction, so called skin tilt pattern and skin slant pattern. The differences associated with the two patterns are estimated as the disruptions in the tilt and slant pattern respectively for lesion classification.
\end{abstract}

Results: Preliminary studies on $11 \mathrm{MM}$ and 28 benign lesions have given Receiver Operating Characteristic (ROC) areas of 0.73 and 0.85 for skin slant and tilt pattern respectively which are better than 0.65 of skin line direction over the same samples.

Conclusions: This paper has demonstrated an important application of 3D skin texture for computer assisted diagnosis of malignant melanoma in vivo. By taking advantage of the extra dimensional information, preliminary studies suggest that some improvements over the existing 2D skin line pattern approach for the differentiation between malignant melanoma and benign lesions.

Key words: 3D skin texture - a reference skin model - 2D Gaussian function - skin tilt pattern - skin slant pattern

\title{
1.0 Introduction
}

Malignant melanoma (MM) is a life-threatening dermatological disease, the successful treatment of which relies heavily on early detection. Employment of a non-invasive automatic analysis method is a useful approach for early differentiation between melanoma and benign pigmented lesions. Conventionally, a group of 2D skin lesion patterns called ABCD (Asymmetry, Border, Color and Diameter) is used as the input to a classification system, however only moderate diagnosis accuracy has been achieved [1]. To obtain better diagnosis results, new parameters which can provide additional information to indicate the presence of melanoma are worth investigating.

Generally, the topography of the skin is directly related to the cell growth patterns under the skin surface. A periodic updating of the skin cells generates a net-like surface with regular geometrical microstructures like triangles and polygons. The important pigmentation cells, melanocytes, may grow up abnormally when the skin receives excessive ultraviolet light radiation or endures some other extreme environment change. Various important skin conditions may present indicators in the form of significant changes in $2 \mathrm{D}$ and $3 \mathrm{D}$ texture. As a typical example, malignant melanoma 
may pathologically occur as a lesion that is variegated in colour and with distortion of the line or net pattern on the skin. Specifically the variable growth rate of the atypical melanocytes not only causes the melanin to be distributed unevenly, resulting in multiple shades of brown or red-brown colour, but it also makes the in-situ lesion exhibit a markedly asymmetrical shape with indentations and protrusions [2]. Therefore, in addition to the pigmentation information which is useful for differentiating melanoma from moles, geometrical 3D microstructure of the skin surface may provide another important clue for evaluation of the skin conditions.

So far, most computer assisted diagnosis systems are based on analysis of photographs of the skin, as photographs still form the majority of accessible dermatological information. It is difficult to capture 3D topography data of the skin due to the dynamic, variegated and multilayered characteristics of the skin. Therefore performing 3D skin texture analysis for diagnosis is still at an early stage.

By extracting a skin texture feature called skin line pattern from macroscopic skin images, malignant melanoma and benign moles can be distinguished through comparison of the line patterns [3][4]. Some new features associated with the skin line pattern like the skin line direction and the skin line intensity have also been found to be useful and complementary to the ABCD features [5]. The skin line patterns extracted from 2D images can only be considered as a kind of pseudo profile of skin surface, as one 2D image is not enough to describe a 3D object completely. The white light clinical images used in the above work are illuminated by side light(s) and are subject to specular and shadow effects. Although multiple illumination processing can reduce the effects, it could also reduce the fine details of skin texture patterns due to an averaging procedure [6].

Handels et al. [7] collected skin 3D profile data by using a high resolution laser profilometer scanning with a silicon skin replica. The traditional 2D image features like co-occurrence matrices, Fourier features and fractal features were used on the 3D data and fused with artificial intelligence algorithms to provide a significant improvement on classification performance. The long scanning time and high price limit the utility of the laser scanning method. Also their research findings are only based on skin replica rather than in-vivo skin.

Previous work [8] has proposed a six light source photometric stereo device that provides a comprehensive description of the skin. It can obtain both 2D surface reflectance and 3D surface normals independent from external environment effects such as ambient illumination. Preliminary evidence suggests that the information obtained should be more suitable than conventional photographs for visual and automated assessment of skin.

Motivated by the observation that skin texture patterns are likely to be disrupted by malignant melanoma and not by benign lesions, this work is aimed at finding the surface disruptions in $3 \mathrm{D}$ rather than $2 \mathrm{D}$ as used in the skin line pattern technique. Rather than analyse the surface topography from reconstruction, the proposed work is seeking to analyse the surface normal vectors directly. As pointed out by Worthington [9], surface normal maps are valid representations for pattern recognitions. The basic idea of the proposed approach is to simulate the surface normals of skin using a standard 2D Gaussian distribution model, and to compare them with a lesion's existing surface normals in the tilt and slant direction. The differences associated with these two directions are defined as the disruptions in skin tilt pattern and skin slant pattern respectively. It is expected that for a malignant melanoma, the differences are larger than for a benign lesion. 
Key words: 3D skin texture - a variable 3D skin model - Gaussian function - skin tilt pattern - skin slant pattern

\subsection{Material and methods}

\subsection{The concept of photometric stereo and some notations}

The surface normal vectors used for analysis in the current study are obtained from a six-light photometric stereo system; its theoretical foundations are briefly outlined here. For an ideal Lambertian surface, the image irradiance equation can be expressed as:

$$
i=\rho \frac{-p \cos \alpha \sin \beta-q \sin \alpha \sin \beta+\cos \beta}{\sqrt{p^{2}+q^{2}+1}}
$$

Where $\alpha$ and $\beta$ are tilt and slant angles of the illuminants, $p=\mathrm{d} z / \mathrm{d} x$ and $q=\mathrm{d} z / \mathrm{d} y$ (where $z$ is the surface height) are the $\mathrm{x}$ axis component and y-axis component of the surface gradients respectively, and $\rho$ is the surface reflection rate (albedo). Although the surface height can be reconstructed through the combination of $p$ and $q$, this process is subject to a number of limitations and can introduce errors, so the effort of the proposed approach will be focused on analysing the 3D surface normals directly.

From Eq. 1, we have

$$
i=\rho(m, n)(\cos \alpha \sin \beta, \sin \alpha \sin \beta, \cos \beta) \cdot \frac{(-p,-q, 1)^{T}}{\sqrt{p^{2}+q^{2}+1}}
$$

where ${ }^{T}$ denotes the transpose.

According to Horn [10],

$$
\vec{N}=\left(n_{x}, n_{y}, n_{z}\right)=\frac{(-p,-q, 1)}{\sqrt{1+p^{2}+q^{2}}}
$$

where $\bar{N}$ is the surface normal vector $n_{x}, n_{y}$ and $n_{z}$ its components in x-axis, y-axis and z-axis respectively. Hence the surface gradient vectors can also be calculated as

$$
p=-\frac{n_{x}}{n_{z}} \text { and } q=-\frac{n_{y}}{n_{z}}
$$

Substituting Eq.3 into Eq.2, we have 


$$
i=\rho(\cos \alpha \sin \beta, \sin \alpha \sin \beta, \cos \beta) \cdot\left(n_{x}, n_{y}, n_{z}\right)^{T}
$$

Since a unit surface normal has the modular of 1 , only three variables are unknown including the albedo and any two from $n_{x}, n_{y}$ and $n_{z}$. So we need at least 3 lights to solve the equation. All other lights have redundant information which can be used for detecting problematic pixels in specular and shadow areas and remove them from the computation of the skin surface normal and reflectance images [11].

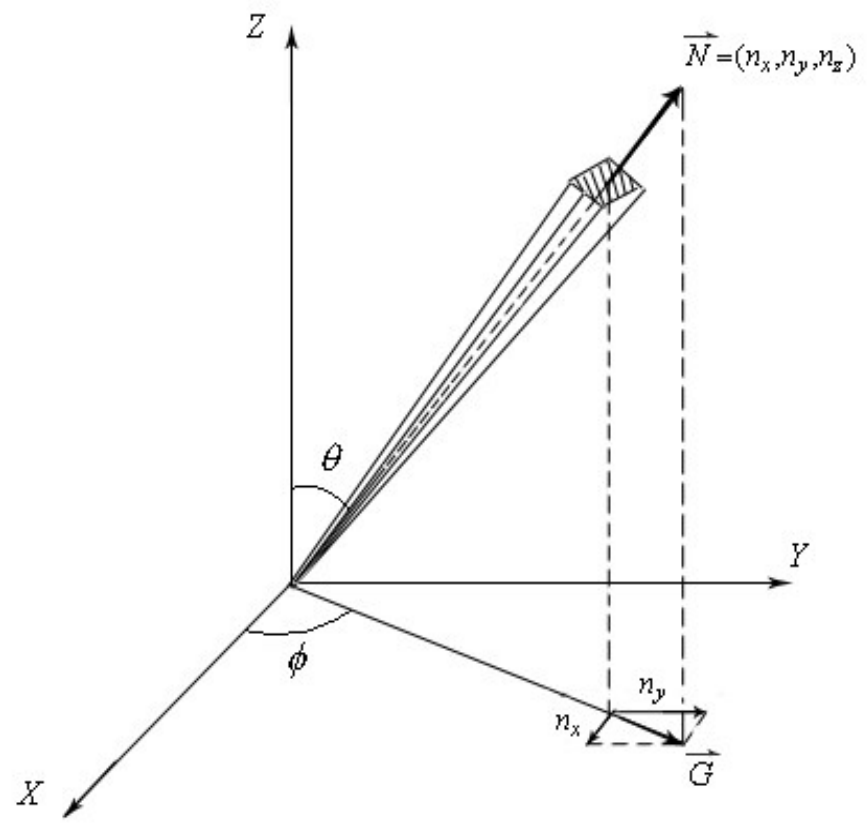

Figure 1 A surface normal vector $\left(n_{x}, n_{y}, n_{z}\right)$ : its tilt direction is $\phi$ and its slant direction $\theta$.

Figure 1 shows that every surface normal on the surface can be identified by its tilt direction $\phi$ and slant direction $\theta$, which can also be represented by surface gradients as

$$
\begin{aligned}
& \phi=\left\{\begin{array}{cc}
\tan ^{-1}\left(\frac{n_{y}}{n_{x}}\right)=\tan ^{-1}\left(\frac{q}{p}\right) & \text { if } n_{x} \geq 0 \text { AND } n_{y} \geq 0 \\
2 \pi+\tan ^{-1}\left(\frac{n_{y}}{n_{x}}\right)=2 \pi+\tan ^{-1}\left(\frac{q}{p}\right) & \text { if } n_{x} \geq 0 \text { AND } n_{y} \leq 0 \\
\pi+\tan ^{-1}\left(\frac{n_{y}}{n_{x}}\right)=\pi+\tan ^{-1}\left(\frac{q}{p}\right) & \text { otherwise }
\end{array}\right. \\
& \theta=\cos ^{-1}\left(n_{z}\right)=\cos ^{-1}\left(\frac{1}{\sqrt{\left(p^{2}+q^{2}\right)+1}}\right)
\end{aligned}
$$

where $\vec{G}$ is the projected vector of the surface normal vector $\vec{N}$ on the image plane. It can be observed that Eq. 6 is made up of the surface gradient direction and Eq. 7 is made up of the surface gradient magnitude and a scalar $n_{z}$. The employment of its tilt direction $\phi$ and slant direction $\theta$ will give more instinctive physical meanings than that of surface gradient or normal. Meanwhile, analysing those 2 quantities is the equivalent of analysing the surface normals themselves directly. Therefore the surface normals in the tilt and slant direction may be referred as skin tilt pattern and skin slant pattern. 


\subsection{D skin texture}

It should be noted here that the concept of surface normals derived from the six-light photometric stereo should not be confused with 2D image gradients. This is because surface normals belong to surface topographic texture (or 3D texture) [12]. It is much different from 2D image texture including the image gradients which inherently are the features formed by pixel intensities under a particular illumination direction, and so is illumination-dependent. This is also the main difference between the 2D skin line pattern and the proposed 3D skin texture. Two examples are shown in Figure 2 where 2D skin line pattern are represented as flows of skin line directions, and 3D skin texture profiles are represented as needle maps of surface gradients, which have a trigonometric relation with surface normals as described in Eq. 4 .
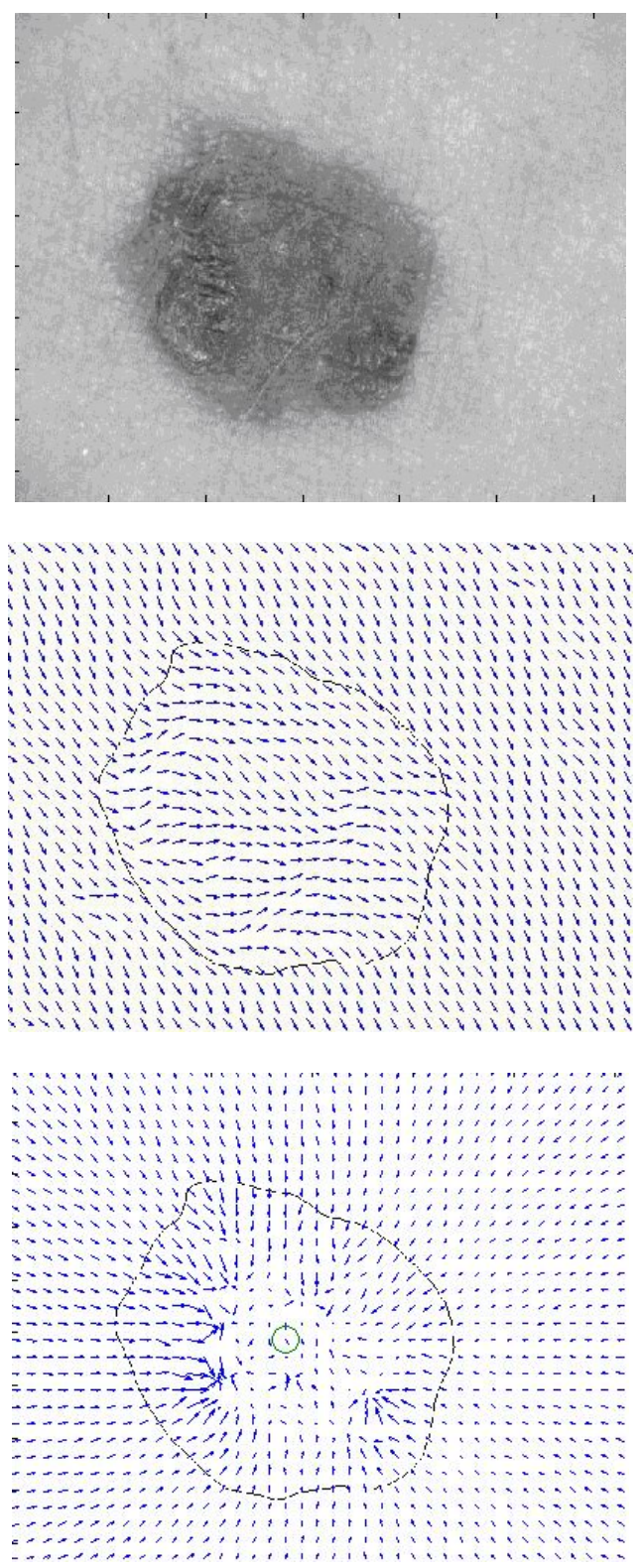
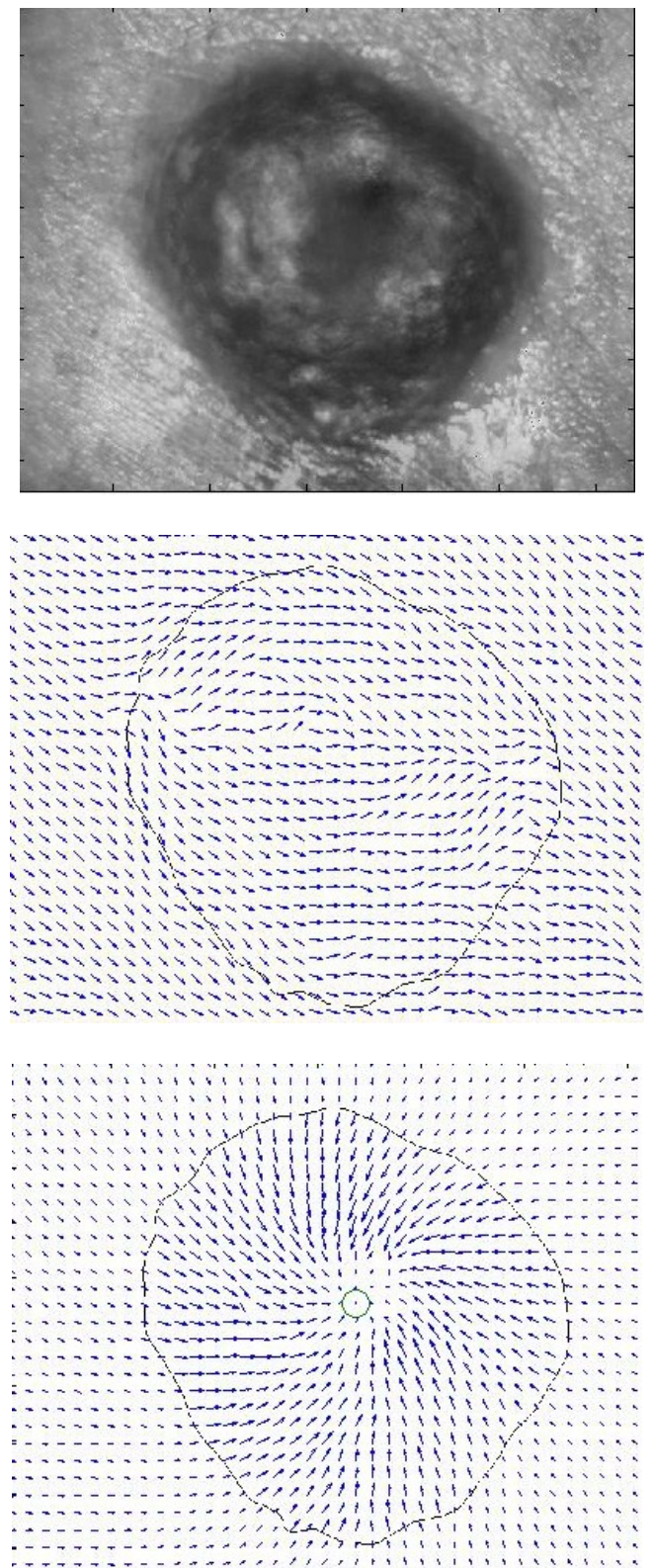

Figure 2 Left row (from top to bottom): a malignant melanoma's conventional photograph, its 2D skin line pattern, its 3D skin texture shown as surface gradient plot; Right row(from top to bottom): a seborrhoeic keratosis's conventional photograph, its 2D skin line pattern, its 3D skin texture shown as surface gradient plot. The distribution centres for both lesions have been marked as ' 0 ' on the surface gradient plots. 


\subsubsection{A variable 3D skin model}

Having obtained the 3D surface normal vectors for each lesion, the next step is to estimate their respective skin disruptions. In order to achieve this, a reference skin model is established, whose surface normals can be used as a reference to be compared with the actual surface normals of the lesion.

Depending on lesion class, a lesion's topography can differ from near flat or slightly raised (such as a benign compound nevus or a junctional nevus) to non-flat or elevated (such as a dome-shaped intradermal nevus). For lesions with flat topography, a skin slant pattern model may be established as a map of constant skin slant patterns. However, this scheme is only feasible for measuring flat lesions but not for non-flat lesions. For non-flat lesions, the underlying surface topography can influence the 3D distribution of surface normals and the skin slant patterns. As a result, it is difficult to distinguish whether the directional differences between the acquired skin slant patterns and the skin slant pattern model are caused by surface topographical variations or indeed caused by disruptions on skin slant patterns due to the abnormal growth of MM.

Figure 3 illustrates this problem. On the left of the figure, the regular textured skin patterns are shown as isosceles triangles and a disrupted skin pattern is shown as a dotted scalene triangle. On the right of the figure, the dotted hemisphere represents a hemispherical-shaped lesion, on which the skin texture patterns are shown as isosceles and are regularly distanced and well-maintained. In this figure, the surface normal vector $\overline{N_{b s}}$ from the hemispherical-shaped lesion has the same direction as the one $\overline{N_{d s}}$ from the disrupted skin pattern. If both are compared with the surface normal $\overline{N_{s}}$ from the reference skin model with constant skin slant patterns, they would yield the same directional difference and may both be recognised as disrupted skin patterns.

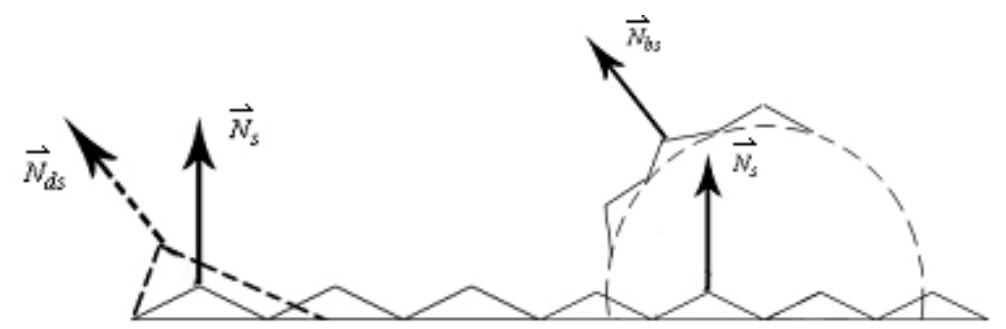

Figure 3 Left: the skin pattern disruption at a pixel is defined as the directional difference between $\overrightarrow{N_{d s}}$ and $\overrightarrow{N_{s}}$ where surface normal of a patch of disrupted skin is shown as $\overline{N_{d s}}$ the surface normal of benign skin is shown as $\overline{N_{s}}$, and the surface normal of benign skin on a spherical lesion is shown as $\overline{N_{b s}}$.

To tackle this problem, this work proposes to use a variable 3D skin model to analyse the 3D skin surface pattern disruptions. The purpose of employing such a 3D variable skin model is to adaptively select a 3D skin model that bestfits a lesion's obtained skin surface patterns from the Skin Analyser. A natural candidate in this category would be a 2D Gaussian function for the reasons explained at the beginning of the paper.

\subsubsection{Disruptions in skin tilt pattern}

The shape of a Gaussian distribution may be denoted as

$$
Z^{*}(u, v)=A^{*} \mathrm{e}^{-\frac{\left(u-u_{c}^{*}\right)^{2}+\left(v-v_{c}^{*}\right)^{2}}{2\left(\sigma^{*}\right)^{2}}}
$$


Where $A^{*}$ is the amplitude, $\left(u_{c}^{*}, v_{c}^{*}\right)$ is the centre and $\sigma^{*}$ is the variance of the Gaussian function (which is equal in both $\mathrm{x}-$ axis and y-axis).

As shown in Eq. 6, the skin tilt pattern is a direction defined entirely within the lesion area on the image plane, so it can be used to determine the centre location of a Gaussian distribution. By projecting the Gaussian envelope onto the image plane, we obtain an isotropic distribution of tilt directions centred at $\left(u_{c}^{*}, v_{c}^{*}\right)$. Regarding an isotropic distribution, as shown by Figure 4, the tilt direction for every point $(u, v)$ of the isotropic distribution can be determined by its distance to the distribution centre $\left(u_{c}^{*}, v_{c}^{*}\right)$ in the $\mathrm{x}$-axis and $\mathrm{y}$-axis. Hence, by specifying the centre of the Gaussian function, we can effectively generate a map of the tilt directions for every point in the image. Since the centre of Gaussian function can be anywhere on the image, we can generate a series of Gaussian envelopes whose number depends on the number of points within the image. In relation to the skin reference model, our objective is to find a surface description that is closest to a lesion, so the centre of the Gaussian function will have to be the one whose Gaussian function best-fits (or more formally, giving the minimum directional differences) a lesion's acquired surface normal distribution from the Skin Analyser. This can be described by the following equation.

$$
\left(u_{c}^{*}, v_{c}^{*}\right)=\arg \min \sum_{\{(u, v)\} \in S_{l}}\left\|\left(\phi(u, v)-\phi^{*}(u, v)\right)\right\|
$$

Where $\left(u_{c}^{*}, v_{c}^{*}\right)$ is the estimated centre of the Gaussian function where the star sign "*” denotes an estimated variable, $S_{l}$ denotes the lesion region, $\|$.$\| denotes the Euclidean distance. \phi$ is the acquired tilt direction and $\phi^{*}$ is the tilt pattern of the Gaussian function. Upon finding this distribution centre, the associated directional differences are defined as the Overall disruptions in skin Tilt pattern (OT) between the skin tilt patterns $\phi_{\min }^{*}$ of the best-fit Gaussian function and the existing skin tilt patterns $\phi$.

$$
\overline{\phi_{\Delta}}=\frac{\sum_{(u, v) \in S_{l}}\left\|\left(\phi_{\min }^{*}(u, v)-\phi(u, v)\right)\right\|}{S_{l}}
$$

\subsubsection{Disruptions in skin slant pattern}

So far, for finding the centre location of the Gaussian distribution, all the computations are limited to the $\mathrm{x}-\mathrm{y}$ plane and the tilt direction. To determine the exact topography of the Gaussian distribution, the slant directions should also be used.

Since the topography of a Gaussian distribution is dependent on its variance and amplitude, by varying the variance and the amplitude, different Gaussian distributions with different topographies are generated. Among them, only the topography or portions of the topography whose skin slant patterns best-fit a lesion's acquired skin slant patterns, was used to quantify the surface disruptions. This can be defined by the following equation

$$
\left(A^{*}, \sigma^{*}\right)=\arg \min \sum_{(u, v) \in S_{l}}\left\|\theta^{*}(u, v)-\theta(u, v)\right\|
$$

where $\theta=\cos ^{-1}\left(n_{z}\right)=\cos ^{-1}\left(\frac{1}{\sqrt{p^{2}+q^{2}+1}}\right)$ and $\theta^{*}=\cos ^{-1}\left(n_{z}^{*}\right)=\cos ^{-1}\left(\frac{1}{\sqrt{\left(Z_{x}^{*}\right)^{2}+\left(Z_{y}^{*}\right)^{2}+1}}\right)$ 
where $p$ and $q$ are the acquired surface gradients (trigonometric variants of 3D surface normals) in the $\mathrm{x}$-axis and $\mathrm{y}$-axis of a lesion from the Skin Analyser and $\left(Z_{x}^{*}, Z_{y}{ }^{*}\right)$ are the surface gradients in the $\mathrm{x}$-axis and $\mathrm{y}$-axis of the Gaussian functions. The Levenberg-Marquardt method [13] was used to estimate the best-fit parameters that solve the nonlinear optimization problem in Eq.11.

Upon determination of $\sigma^{*}$ and the resultant Gaussian topography or portions of the Gaussian topography, the associated differences are used to define the degree of disruptions in skin slant pattern. The Overall disruptions in skin Slant pattern (OS) is defined as the sum of the directional differences between the skin slant patterns $\theta_{\min }^{*}$ of best-fit Gaussian function and the acquired skin slant patterns $\theta$.

$$
\overline{\theta_{\Delta}}=\frac{\sum_{(u, v) \in S_{l}}\left\|\theta_{\min }^{*}(u, v)-\theta(u, v)\right\|}{S_{l}}
$$

In the following experiment, the proposed two features including the skin tilt pattern feature (or OT in Eq. 10) and the skin slant pattern feature (or OS in Eq. 12) will be used for lesion classification. To tackle the false "disruptions" caused by noise, the skin tilt pattern and skin slant pattern disruptions are both enhanced by 3 iterations of median filters with the window size of $3 \times 3$. The discriminating power of the proposed two pattern features will be compared with the best $2 \mathrm{D}$ skin line pattern feature [4][5], Skin Line Direction (SLD) over the same sample set. The reason for comparing with the $2 \mathrm{D}$ skin line direction is because it is the best single 2D feature (among others which include the classic $\mathrm{ABCD}$ features and skin line intensity feature) according to [5].

\subsection{Results}

The experiments are carried out on 39 lesions of which 11 are MMs. The 28 benign lesions consist of 8 classes including 2 Dermatofibromas (DF), 4 Intradermal nevi (IN), 2 hyperkeratotic squamous papillomas (HSP), 8 compound nevi (CPN), 7 seborrhoeic keratoses (SK)s, 2 dysplastic nevi (DN), 2 congenital nevi (CGN) and 1 junctional nevus (JN). All of these lesions were captured at the collaborating institutes using the skin analyser.

The 28 benign lesions are subsequently split into 4 sample groups as listed in Table 1. Sample group 1 includes $1 \mathrm{JN}$ and $8 \mathrm{CN}$ based on the fact that both lesions are typically small, smooth and slightly raised. Sample group 2 includes 7 SKs which are among the most common classes of benign lesions and have a distinct appearance from other benign lesions, sample group 3 includes 4 IN and 2 DFs based on the fact that they both have raised and nodular shape. Sample group 4 is made up of the rest of the benign lesions including 2 DN, 2 CGN, 2 HSPs and 1 blue nevus. 
The scatter plots for the skin tilt pattern and skin slant pattern are shown in Figure 5 and Figure 6 respectively. Using the same data samples, the scatter plot for the skin line direction based on [4] is illustrated in Figure 7. The discriminating power of each feature is judged by the area under the Receiver-Operating-Characteristic (ROC) curve or ROC area. The ROC curves for different groups of benign lesions versus the same $11 \mathrm{MMs}$ are shown in Figure 8. The corresponding ROC results are shown in Table 2.

As can be seen from Table 2, the overall ROC areas for skin tilt pattern and skin slant pattern for all classes of lesions are 0.73 and 0.85 respectively, both are better than 0.65 achieved by the skin line direction. Although this ROC result for skin line direction is not as high as the one reported by [5], it should be noted that only two types of benign lesions, i.e. $\mathrm{JN}$ and CPN, are used in their experiment. In the current experiment, for sample group 1 which consists of JN and CPN, the result for skin line direction improves to 0.80; a figure in parallel with the one reported by [5] and is equally best with the skin slant feature. Apart from this sample group, the skin slant pattern feature also shows the best results for sample group 2, sample group 3 and all lesion samples. This is probably because skin slant pattern is defined in the Z-axis direction, which can reveal extra dimensional information. In comparison, skin line direction is inherently a 2D feature while skin tilt pattern is entirely defined on the $2 \mathrm{D} x-y$ plane.

It can be seen that from Table 2, the classification results for both the skin slant pattern feature and the skin line direction for sample group 4 which consists of 2 DN, 2 CGN and 2 HSPs are worse than all the other sample groups, this is not surprising as the high difficulty of classifying dysplastic nevi as benign lesions have been generally acknowledged. In fact, the DN and CGN used in the experiments have even been mistaken by the experienced dermatologists at the collaborating clinics and all have subsequently been sent for biopsies. Still substantial improvements over the skin line pattern technique are achieved by the proposed two pattern features with a promising result of 0.83 for the skin tilt pattern feature and 0.77 for the skin slant pattern feature.

\subsection{Discussion}

Prior work using the skin line pattern technique for the diagnosis of malignant melanoma has used a conventional 2D approach. The classification performance thereby obtained for separating junctional and compound nevi from malignant melanoma have in fact been promising. However, the approach has 3 weaknesses which should be considered. Firstly, the classification performance relies heavily on obtaining accurate lesion intensities. According to the nature of lesion reflectance, lighting distortions such as specular reflections and shadows are not uncommon. In many cases, these lighting distortions can affect skin line recovery and the classification performance. Secondly, because the computation method [4] for calculating skin line direction used signed average, it is subject to cancelling-out effects, which will make the classification hard to comprehend. This is evident in the more recent skin line pattern publication [14], where only part of the lesion is used for classification which potentially can reduce these cancelling-out effects. Thirdly, from the generality point of view, the recovery of skin line patterns is sensitive to the value for the $9 \times 9$ high-pass filtering scheme used at the beginning for smoothing the skin photographs. With different values (or bandwidths more formally) for smoothing, it is likely that distinct skin line patterns will be generated that could affect the subsequent classification performance. 
The proposed statistical analysis on the 3D skin texture does not have the same problems for the following reasons. Firstly, it takes advantages of the additional light sources and images in the photometric stereo technique to isolate lighting/viewing distortions and improve classification performance. Secondly, the idea of the proposed approach is to use Gaussian functions to simulate a series of reference skin models, and to find the reference skin model that best-fits (or closest to) the surface normal distribution of a lesion. Euclidean distance is used to measure these directional differences and there isn't a cancelling-out problem. Third, parameters used in the proposed 3D texture analysis are not set before doing the experiments, those parameters include the centre $\left(u_{c}^{*}, v_{c}^{*}\right)$, the amplitude $A$ and the variance $\sigma$ for Gaussian functions. They are found through two separate optimisation procedures (in skin tilt and slant pattern respectively) which minimise the anisotropic differences between the Gaussian functions and the actual surface normal distributions of a lesion.

However, the fundamental difference between the skin line pattern approach and the proposed approach still lies on the difference between 2D skin texture and 3D skin texture. This is because the former one reflects pixel intensity features, while the latter one reflects the inherent objectivity of a lesion. Therefore the proposed analysis of 3D skin texture is independent of the existing 2D techniques, so it provides a new and distinct method for MM diagnosis.

\subsection{Conclusions}

This paper has demonstrated an important application of 3D skin texture for computer assisted diagnosis of malignant melanoma in vivo. By taking advantage of the extra dimensional information, preliminary studies suggest that improvements are possible over the existing 2D skin line pattern approach for separating malignant melanoma from benign lesions. In terms of methodology, the proposed approach employs a parametric technique i.e. Gaussian function to adaptively model the surface topographical characteristics of both spherical lesions and flat lesions. Whether a reference skin model derived from non-parametric statistical models can improve the classification performance, will be investigated in future work.

\section{References}

[1]. Sboner A, Eccher C, Blanzieri E, Bauer P, Cristofolini M, Zumiani G, Forti SA. Multiple classifier system for early melanoma diagnosis", Artif Intell Med. 2003: 27(1): 29-44.

[2]. Lee TK. Measuring border irregularity and shape of cutaneous melanocytic lesions. PhD thesis of Simon Fraser University 2001.

[3]. Round AJ, Duller AWG and Fish PJ. Lesion classification using skin patterning. Skin research and technology 2000: 6(4): 183192.

[4]. She Zand Fish PJ, Analysis of skin line pattern for lesion classification, Skin research and technology 2003: 9(1): 73-80.

[5]. She Z, Liu Y, Damato A. Combination of features from skin pattern and ABCD analysis for lesion classification. Skin Research and Technology 2007: 13(1): 25-33.

[6]. She Z, Dickson M, Fish PJ, Duller AWG, Bamber JC. Specular reflection reduction in context of skin line recovery. Proceedings of Medical Image and Understanding and Analysis 2001: 121-124.

[7]. Handels H, Ross T, Kreusch J, Wolff HH Poppl SJ. Feature selection for optimized skin tumor recognition using genetic algorithms. Artif. Intell. Med. 1999: 16(3): 283-297. 
[8]. Sun J, Smith ML, Smith LN, Coutts L, Dabis R, Harland C, Bamber J, Reflectance of human skin using colour photometric stereo: with particular application to pigmented lesion analysis. Skin Research and Technology 2008: 14 (2): 173-179.

[9]. Worthington PL, Hancock ER. New Constraints on Data Closeness and Needle-map Consistency for Shape-from-Shading. IEEE Transactions on Pattern Analysis and Machine Intelligence 1999: 21: 1240--1267.

[10].Horn BKP. Robot Vision 1986: MIT press.

[11].Sun J, Smith ML, Smith LN, Midha S, Bamber J. Object surface recovery using a multi-light photometric stereo technique for non-Lambertian surfaces subject to shadows and specularities. Image and Vision Computing 2007: 25: 1050-1057.

[12].Smith ML, Farooq AR, Smith LN Midha S. Surface texture inspection using conventional techniques applied to a photometrically acquired bump map. Sensor review 2000: 20(4): 299-307.

[13]. Gill P, Murray W and Wright M, Practical Optimization 1981: Academic Press.

[14].She Z, Liu Y, Tatler J, Skin pattern analysis for lesion classification using gradient of skin line direction, Proceedings of Medical Image Understanding and Analysis 2007: 46-50.

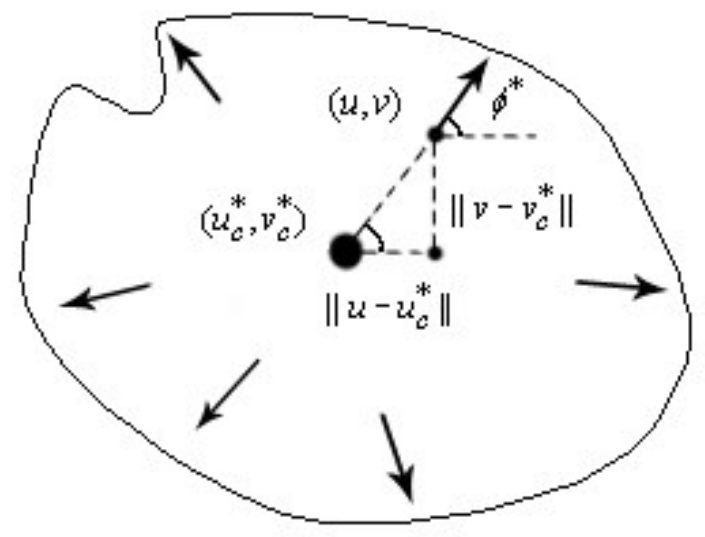

Figure 4 The tilt direction at a point $(u, v)$ can be determined by its distance to the distribution centre $\left(u_{c}^{*}, v_{c}^{*}\right)$ in $\mathbf{x}$-axis and $\mathbf{y}$ axis, hence it can be calculated as $\phi^{*}=\tan ^{-1}\left(\left\|v-v_{c}^{*}\right\| /\left\|u-u_{c}^{*}\right\|\right)$ where $\|\cdot\|$ denotes the Euclidean distance.

\begin{tabular}{|c|c|c|c|}
\hline Sample group & Lesion class & Number in class & Total \\
\hline \multirow[t]{2}{*}{ Group 1} & Compound nevus & 8 & \multirow[t]{2}{*}{9} \\
\hline & Junctional nevus & 1 & \\
\hline Group 2 & Seborrheic Keratosis & 7 & 8 \\
\hline \multirow[t]{2}{*}{ Group 3} & Intradermal nevus & 4 & \multirow[t]{2}{*}{6} \\
\hline & Dermatofibroma & 2 & \\
\hline \multirow[t]{3}{*}{ Group 4} & Hyperkeratotic Squamous Papillomas & 2 & \multirow[t]{3}{*}{6} \\
\hline & Congenital nevus & 2 & \\
\hline & Dysplastic nevus & 2 & \\
\hline
\end{tabular}

Table 1 Benign lesions are split into 4 sample groups with benign lesion classes 


\begin{tabular}{|c|c|c|c|c|}
\hline & graphs in Figure 6 & & & \\
\hline Group 1 & (a1-c1) & 0.77 & 0.80 & 0.80 \\
\hline Group 2 & (a2-c2) & 0.47 & 0.84 & 0.52 \\
\hline Group 3 & $(\mathrm{a} 3-\mathrm{c} 3)$ & 0.90 & 0.97 & 0.80 \\
\hline Group 4 & $(\mathrm{a} 4-\mathrm{c} 4)$ & 0.83 & 0.77 & 0.33 \\
\hline All samples & $(\mathrm{a} 5-\mathrm{c5})$ & 0.73 & 0.85 & 0.65 \\
\hline
\end{tabular}

Table 2 The corresponding ROC areas for the ROC curves in Figure 8

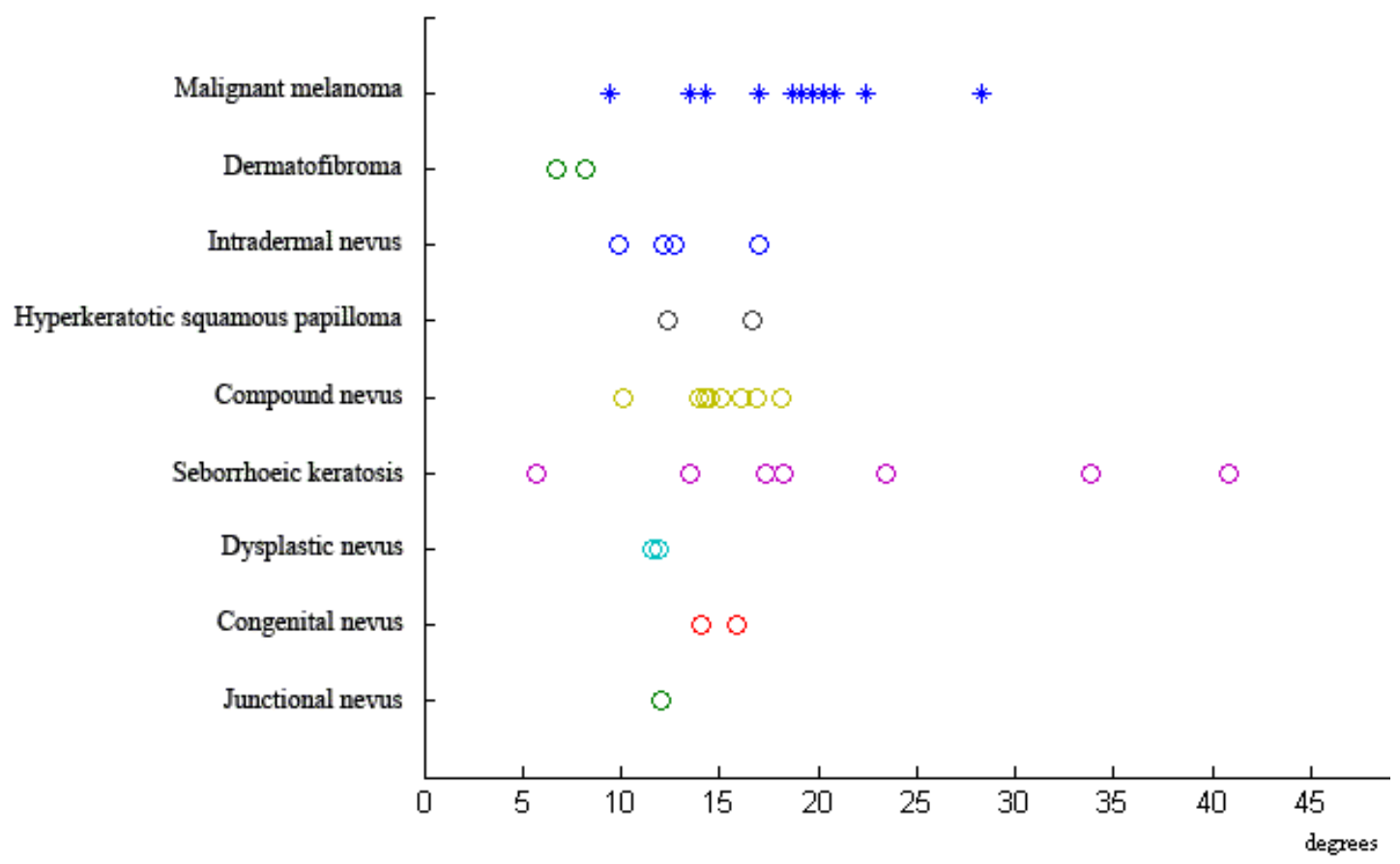

Figure 5 Scatter plot for the overall disruptions in skin tilt pattern (OT)

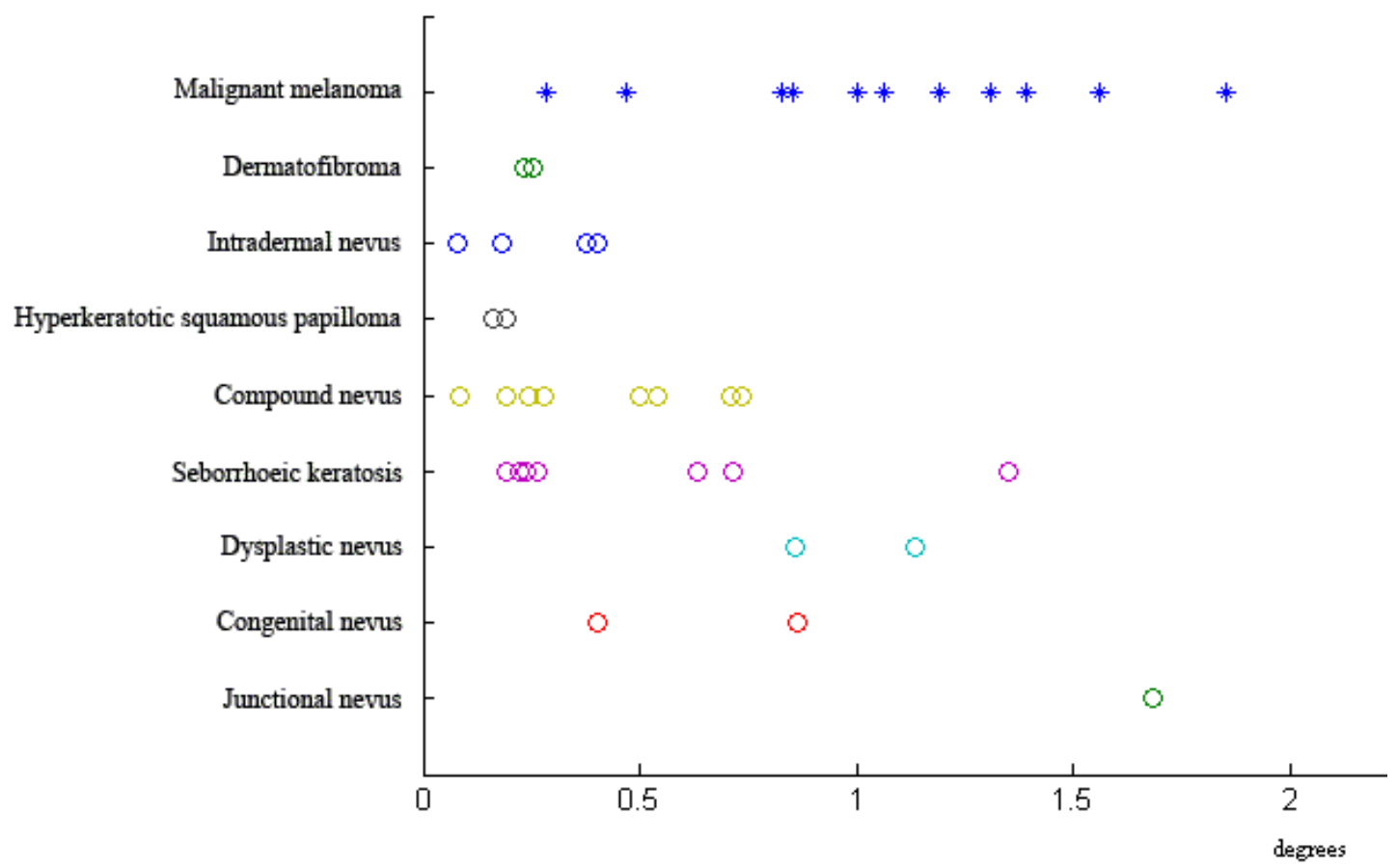


Figure 6 Scatter plot for the overall disruptions in skin slant pattern (OS)

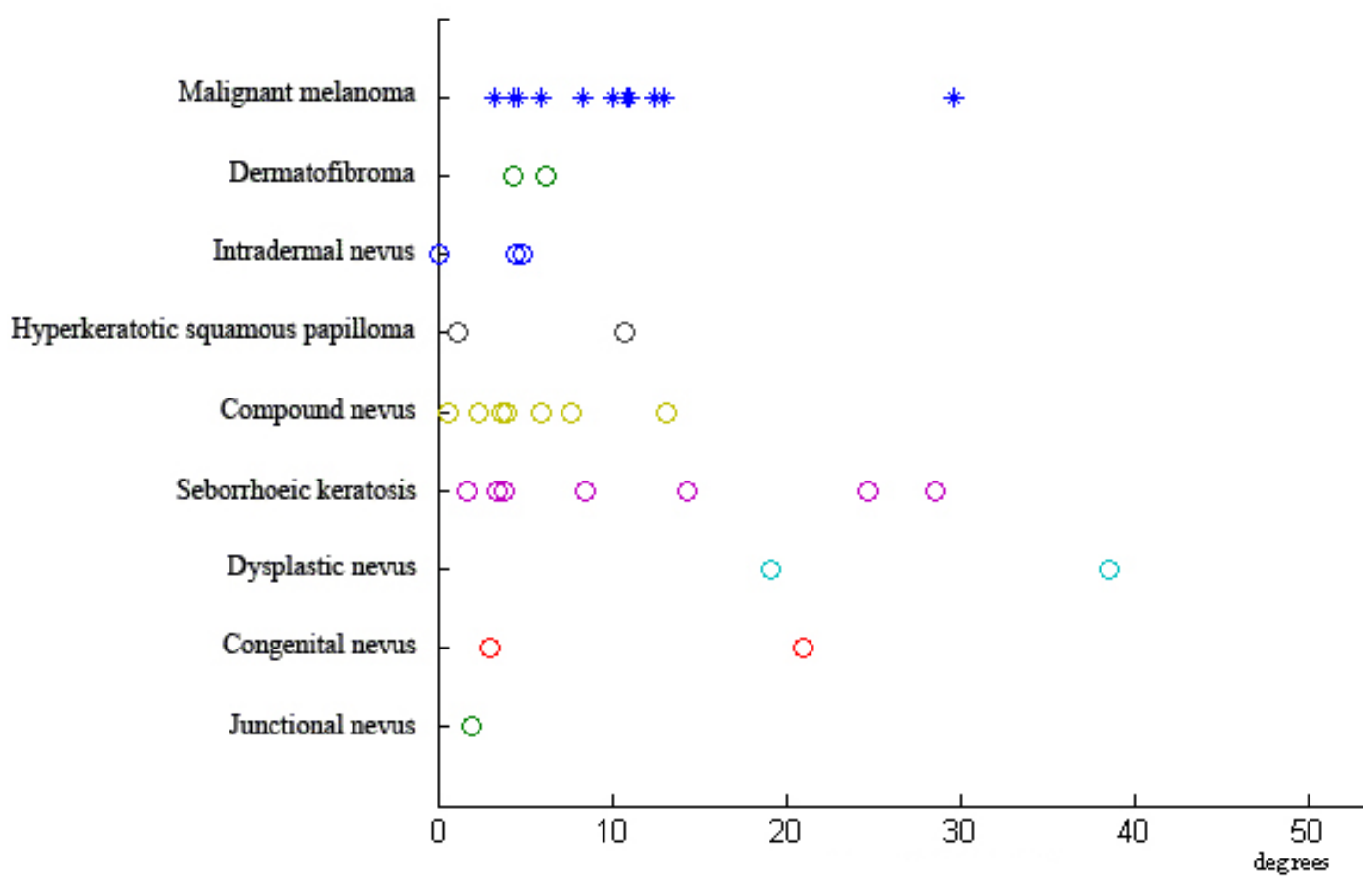

Figure 7 Scatter plot for features from the skin line direction 


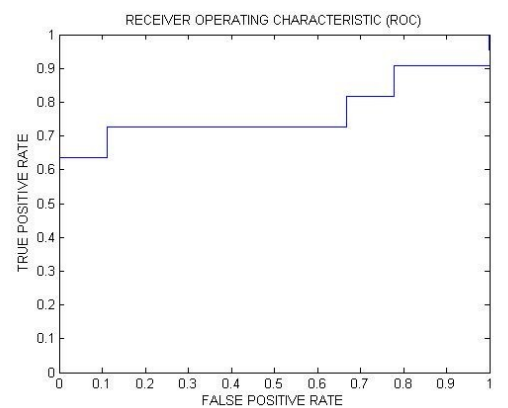

(a1)

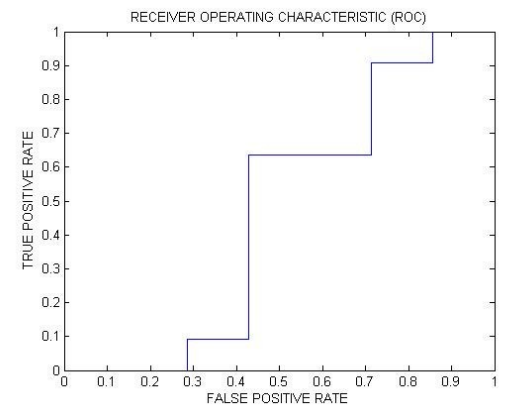

(a2)

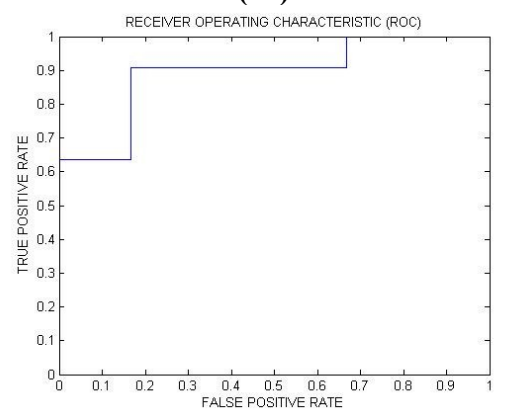

(a3)

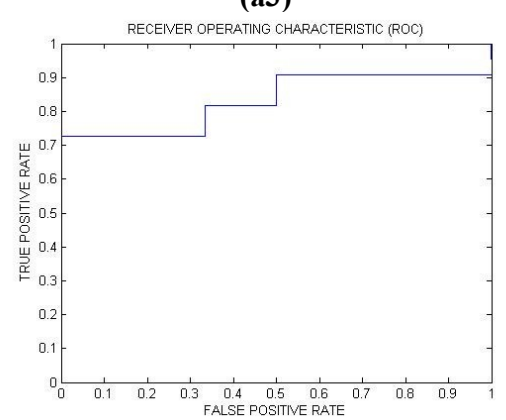

(a4)

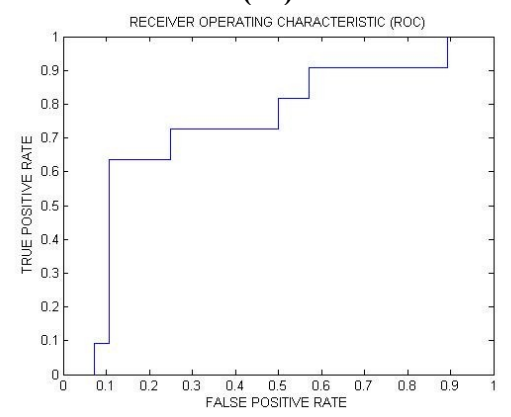

(a5)

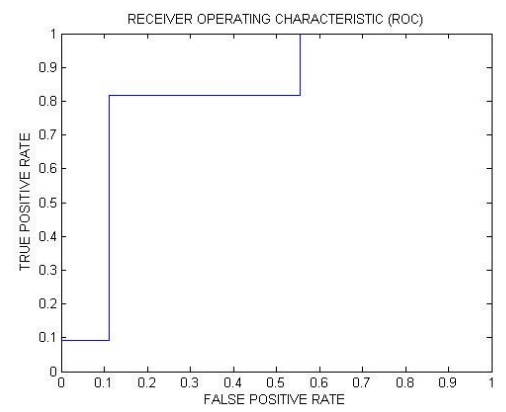

(b1)

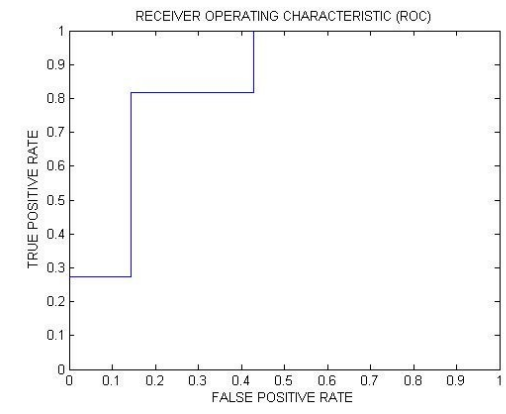

(b2)

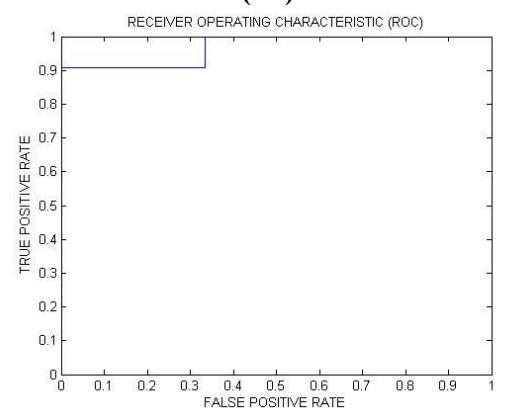

(b3)

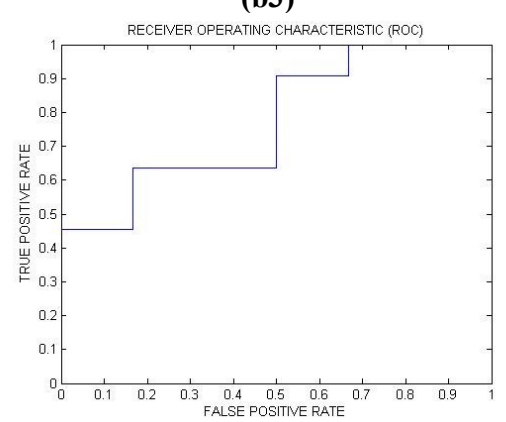

(b4)

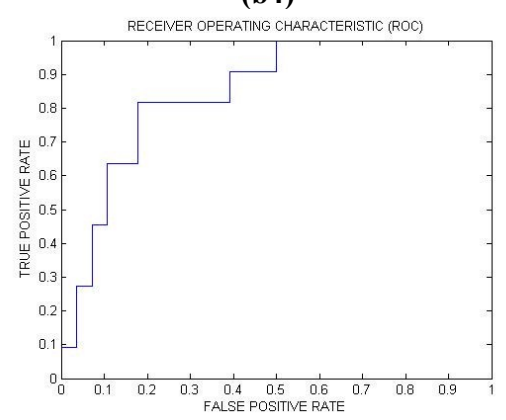

(b5)

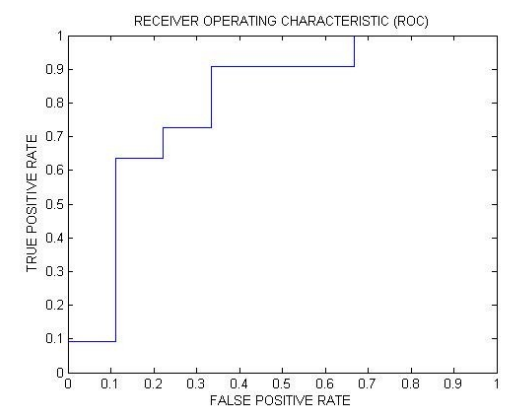

(c1)

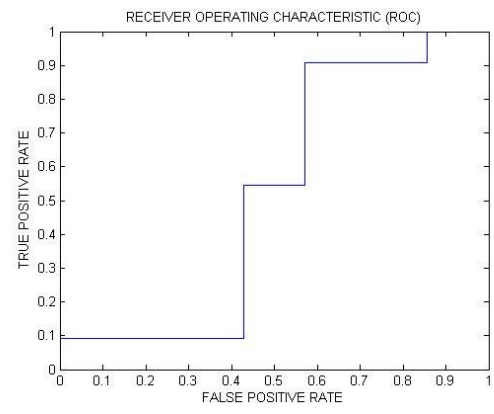

(c2)

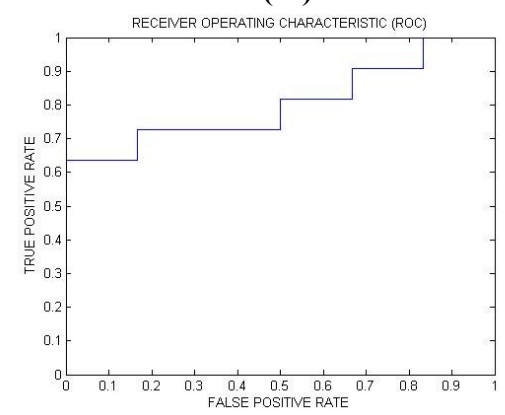

(c3)

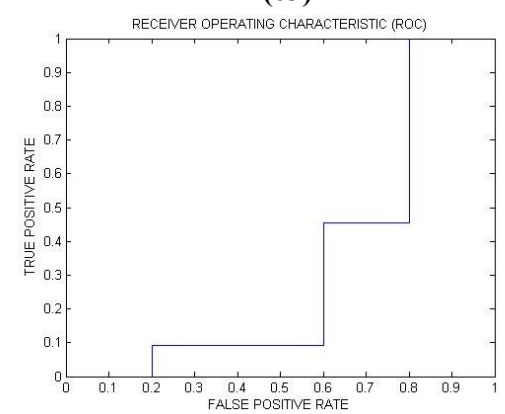

(c4)

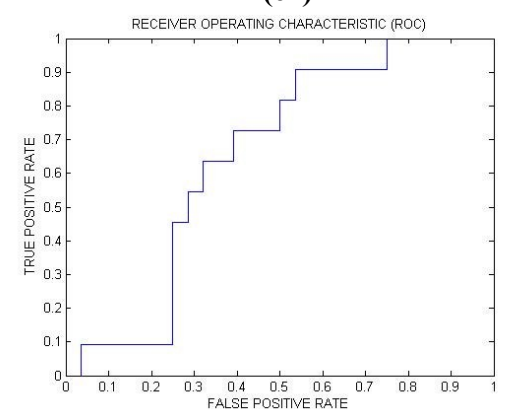

(c5)

Figure 8 Receiver-Operating-Characteristic (ROC) curves for skin tilt pattern (a1-a5), skin slant pattern (b1-b5) and skin line direction (c1-c5); The ROCs are generated from different sample groups of benign lesions against the same 11 MMs. The ROC curves for sample group 1 (a1-c1), for sample group 2 (a2-c2), sample group 3 (a3-c3), sample group 4 for (a4-c4) and all lesion samples for (a5-c5). 